\title{
NONUNIQUENESS FOR THE RADON TRANSFORM
}

\author{
D. H. ARMITAGE AND M. GOLDSTEIN
}

(Communicated by J. Marshall Ash)

\begin{abstract}
There exists a nonconstant harmonic function $h$ on $\mathbb{R}^{N}$, where $N \geq 2$, such that $\int_{P}|h|<+\infty$ and $\int_{P} h=0$ for every $(N-1)$-dimensional hyperplane $P$.
\end{abstract}

Let $f$ be a real- or complex-valued function on $\mathbb{R}^{N}(N \geq 2)$, and suppose that $f$ is integrable on each $(N-1)$-dimensional hyperplane $P$ in $\mathbb{R}^{N}$. The Radon transform $\widehat{f}$ of $f$ is defined on the set $\mathbb{P}^{N}$ of all such hyperplanes by $\widehat{f}(P)=\int_{P} f d \lambda$, where $\lambda$ denotes $(N-1)$-dimensional Lebesgue measure on $P$. We refer to Helgason [4] for the general theory of the Radon transform and its applications.

There are several proofs that if $f$ is continuous and integrable on $\mathbb{R}^{N}$ and $\widehat{f} \equiv 0$ on $\mathbb{P}^{N}$, then $f \equiv 0$ on $\mathbb{R}^{N}$ (see Zalcman [5] for references); the simplest proof proceeds by showing that, under the stated hypotheses, the Fourier transform of $f$ vanishes identically. With $N=2$, at least, the hypothesis that $f$ is integrable on $\mathbb{R}^{N}$ cannot be removed. Indeed, identifying $\mathbb{R}^{2}$ with $\mathbb{C}$, Zalcman $[5, \S 5]$ showed that there exists a nonconstant entire function $\phi$ such that $\hat{\phi} \equiv 0$ on $\mathbb{P}^{2}$. The real part $h$ of $\phi$ provides an example of a nonconstant harmonic function on $\mathbb{R}^{2}$ such that $\hat{h} \equiv 0$ on $\mathbb{P}^{2}$. Zalcman's proof depends on an approximation theorem of Arakelian [1, p. 1189] for holomorphic functions and has no obvious generalization to $\mathbb{R}^{N}(N \geq 3)$. Here we use a recent theorem [3, Theorem 1.1] on harmonic approximation to prove the following result.

Theorem. There exists a nonconstant harmonic function $h$ on $\mathbb{R}^{N}(N \geq 2)$ such that $\widehat{h} \equiv 0$ on $\mathbb{P}^{N}$.

To the best of our knowledge, it has not hitherto been decided whether there exists even a nonconstant continuous function $f$ on $\mathbb{R}^{N}(N \geq 3)$ for which $\widehat{f} \equiv 0$ on $\mathbb{P}^{N}$.

We denote a typical point of $\mathbb{R}^{N}$ by $x=\left(x_{1}, \ldots, x_{N}\right)$ and write

$$
\langle x, y\rangle=x_{1} y_{1}+\cdots+x_{N} y_{N}, \quad\|x\|=\sqrt{\langle x, x\rangle} \quad\left(x, y \in \mathbb{R}^{N}\right) .
$$

Received by the editors December 17, 1990 and, in revised form, May 22, 1991.

1991 Mathematics Subject Classification. Primary 44A12; Secondary 31B05.

Key words and phrases. Radon transform, harmonic function. 
Let $S$ denote the sphere $\left\{y \in \mathbb{R}^{N}:\|y\|=1\right\}$. If $y \in S$ and $t \in \mathbb{R}$, we write

$$
P(y, t)=\left\{x \in \mathbb{R}^{N}:\langle x, y\rangle=t\right\} ;
$$

if $-\infty \leq a<b \leq+\infty$, we put

$$
Q(y, a, b)=\bigcup_{a<t<b} P(y, t) .
$$

Thus $P(y, t)$ is an $(N-1)$-dimensional hyperplane and $Q(y, a, b)$ is isometric to $\mathbb{R}^{N-1} \times(a, b)$. The point at infinity of $\mathbb{R}^{N}$ is denoted by $\mathscr{A}$, and we understand $\mathbb{R}^{N} \cup\{\mathscr{A}\}$ to be equipped with the Aleksandroff one-point compactification topology.

To prove the theorem, we need a nonempty subset $E$ of $\mathbb{R}^{N}$ with the following properties:

(i) $E$ is open in $\mathbb{R}^{N}$;

(ii) $E \cup\{\mathscr{A}\}$ is connected and locally connected in the topology of $\mathbb{R}^{N} \cup$ $\{\mathscr{A}\}$

(iii) if $y \in S$ and $0<a<+\infty$, then $E \cap Q(y,-a, a)$ is bounded;

(iv) if $y \in S$ then there exists a positive number $T$, depending on $y$, such that at least one of the sets $E \cap Q(y,-\infty,-T)$ and $E \cap Q(y, T,+\infty)$ is empty.

An example of such a set $E$ is as follows. Let $I=[0,+\infty)$, define $\psi: I \rightarrow$ $\mathbb{R}^{N}$ by $\psi(\xi)=\left(\xi, \xi^{2}, \ldots, \xi^{N}\right)$, and put

$$
E=\left\{x \in \mathbb{R}^{N}: \inf _{\xi \in I}\|x-\psi(\xi)\|<1\right\} .
$$

We owe this example to a remark of Dr. T. B. M. McMaster; it replaces a more complicated example of ours. It is clear that the set $E$ defined by (1) has properties (i) and (ii); we verify at the end of this note that it also has properties (iii) and (iv).

Now fix a point $z$ of a set $E$ satisfying (i)-(iv) and define closed subsets of $\mathbb{R}^{N}$ by

$$
F_{1}=\mathbb{R}^{N} \backslash E, \quad F_{2}=\{z\}, \quad F=F_{1} \cup F_{2} .
$$

Clearly $F$ is unbounded. Let $\omega_{1}$ and $\omega_{2}$ be disjoint open subsets of $\mathbb{R}^{N}$ containing $F_{1}$ and $F_{2}$, respectively, and define a function $u$ to be equal to 0 on $\omega_{1}$ and equal to 1 on $\omega_{2}$. Then $u$ is harmonic on the open neighbourhood $\omega_{1} \cup \omega_{2}$ of $F$. Also, properties (i) and (ii) hold with $\mathbb{R}^{N} \backslash F=E \backslash\{z\}$ in place of $E$. It follows from [3, Theorem 1.1] that there exists a harmonic function $h$ on $\mathbb{R}^{N}$ such that

$$
|h(x)-u(x)|<(1+\|x\|)^{-N-1} \quad(x \in F) .
$$

In particular, $|h(z)-1|<1$ and $\lim _{x \rightarrow \mathscr{A}, x \in F} h(x)=0$, so that $h$ is nonconstant.

Let $y$ be a point of $S$. It suffices to show that $h$ is integrable on $P(y, t)$ and $\widehat{h}(P(y, t))=0$ for all real $t$. Suppose that $0<a<+\infty$. By property (iii), we have for some positive number $r$

$$
Q(y,-a, a) \backslash F \subset\left\{x \in \mathbb{R}^{N}:\|x\|<r\right\}=B_{N}(r), \quad \text { say. }
$$


From this remark and (2) we obtain that when $|t|<a$

$$
\begin{aligned}
\int_{P(y, t)}|h| d \lambda & \leq \sup _{B_{N}(r)}|h| \int_{P(y, t) \backslash F} d \lambda+\int_{P(y, t) \cap F}(1+\|x\|)^{-N-1} d \lambda(x) \\
& \leq V(r) \sup _{B_{N}(r)}|h|+\int_{P(y, 0)}(1+\|x\|)^{-N-1} d \lambda(x),
\end{aligned}
$$

where $V(r)$ is the $(N-1)$-dimensional volume of $B_{N-1}(r)$. Thus the function $t \rightarrow \int_{P(y, t)}|h| d \lambda$ is locally bounded on $\mathbb{R}$. Now, using a rotation of axes, we find from known results (see, e.g., [2, Theorem 2]) that if $s$ is subharmonic on $\mathbb{R}^{N}$ and the function $t \rightarrow \int_{P(y, t)}|s| d \lambda$ is locally bounded on $\mathbb{R}$, then the hyperplane mean $\hat{s}(P(y, t))$ is a convex function of $t$ on $\mathbb{R}$. Applying this result with $s=h$ and with $s=-h$, we obtain that $\hat{h}(P(y, t))$ is a linear function (i.e., a polynomial of degree at most 1) of $t$. By property (iv), there exists a positive number $T$ such that $P(y, t) \subset F$ either for all $t>T$ or for all $t<-T$. Also, when $P(y, t) \subset F$ we obtain from (2) that

$$
\begin{aligned}
|\widehat{h}(P(y, t))| & <\int_{P(y, t)}(1+\|x\|)^{-N-1} d \lambda(x) \\
& =\int_{P(y, 0)}\left(1+\sqrt{ }\left(\|x\|^{2}+t^{2}\right)\right)^{-N-1} d \lambda(x) \\
& <(1+|t|)^{-1} \int_{P(y, 0)}(1+\|x\|)^{-N} d \lambda(x),
\end{aligned}
$$

so that $\widehat{h}(P(y, t)) \rightarrow 0$ either as $t \rightarrow+\infty$ or as $t \rightarrow-\infty$. Since $\widehat{h}(P(y, t))$ is a linear function of $t$, it now follows that $\widehat{h}(P(y, t))=0$ for all real $t$.

It remains to verify that the set $E$ given by (1) has properties (iii) and (iv). Fix a point $y$ of $S$ and define $\eta: I \rightarrow \mathbb{R}$ by

$$
\eta(\xi)=\sum_{j=1}^{N} y_{j} \xi^{j} .
$$

Note that $|\eta(\xi)| \rightarrow+\infty$ as $\xi \rightarrow+\infty$ and that $\eta$ is either bounded above or bounded below on $I$. For each point $x$ of $E$, there exist a number $\xi_{x}$ in $I$ and a point $x^{\prime}$ of $B_{N}(1)$ such that $x=\psi\left(\xi_{x}\right)+x^{\prime}$. Clearly $\xi_{x} \rightarrow+\infty$ as $x \rightarrow \mathscr{A}(x \in E)$. We have

$$
\begin{aligned}
\langle x, y\rangle & =\left\langle\psi\left(\xi_{x}\right), y\right\rangle+\left\langle x^{\prime}, y\right\rangle \\
& =\eta\left(\xi_{x}\right)+O(1) \quad(x \rightarrow \mathscr{A}, x \in E) .
\end{aligned}
$$

It follows that if $0<a<+\infty$ then $\{x \in E:|\langle x, y\rangle|<a\}$ is bounded, so that (iii) holds. It also follows that there exists a positive number $T$ such that either $\langle x, y\rangle<T$ for all $x$ in $E$ or $\langle x, y\rangle>-T$ for all $x$ in $E$, so that (iv) holds.

\section{REFERENCES}

1. N. U. Arakelian, Uniform approximation on closed sets by entire functions, Izv. Akad. Nauk SSSR Ser. Mat. 28 (1964), 1187-1206.

2. D. H. Armitage and S. J. Gardiner, The growth of the hyperplane mean of a subharmonic function, J. London Math. Soc. (2) 36 (1987), 501-512. 
3. D. H. Armitage and M. Goldstein, Better than uniform approximation on closed sets by harmonic functions with singularities, Proc. London Math. Soc. (3) 60 (1990), 319-343.

4. S. Helgason, The Radon transform, Birkhäuser, Boston, Basel, and Stuttgart, 1980.

5. L. Zalcman, Uniqueness and nonuniqueness for the Radon transform, Bull. London Math. Soc. 14 (1982), 241-245.

Department of Pure Mathematics, The Queen's University of Belfast, Belfast BT7 1NN, NORTHERN IRELAND

E-mail address: d.armitage@uk.ac.qub.v2

Department of Mathematics, Arizona State University, Tempe, Arizona 85287 